\title{
Analysis of detection results of thyroid function-related indexes in pregnant women and establishment of the reference interval
}

\author{
QI ZHOU $^{1 *}$, YANLI ZHANG $^{1 *}$, JIANHUA ZHOU $^{2}$, XIANGRONG YANG $^{1}$, \\ YUQIANG HUANG ${ }^{1},{\text { HUAFENG } \mathrm{LI}^{1} \text { and CHUNLING MA }}^{3}$ \\ ${ }^{1}$ Clinical Laboratory Center, Women and Children's Health Care Hospital of Linyi; \\ ${ }^{2}$ Department of Neonatology, Women and Children's Health Care Hospital of Linyi, Linyi, Shandong 276002; \\ ${ }^{3}$ Clinical Laboratory Department, Shandong Medical College, Linyi, Shandong 276000, P.R. China
}

Received May 16, 2018; Accepted November 15, 2018

DOI: 10.3892/etm.2018.7135

\begin{abstract}
Detection results of free thyroxine (FT4), thyroid stimulating hormone (TSH) and thyroid peroxidase antibody (TPOAb) in pregnant women in Linyi region were investigated. A total of 22,235 healthy pregnant women admitted to Women and Children's Health Care Hospital of Linyi from October 2016 to October 2017 were selected and divided into the first-trimester, second-trimester and third-trimester pregnancy groups. Non-pregnant women in the same period were selected as the control group. Roche E601 electrochemiluminescence apparatus was applied to detect FT4, TSH and TPOAb, and statistical analysis was then carried out for the detected data. The positive rate in the third trimester of pregnancy was obviously higher than those in the first and second trimesters of pregnancy $(\mathrm{P}<0.05)$. With the prolongation of pregnancy, the reference interval of FT4 was gradually decreased, among which the intervals in the second and third trimesters of pregnancy were significantly lower than those in the non-pregnancy $(\mathrm{P}<0.05)$. With the prolongation of pregnancy, the interval in the first trimester of pregnancy was markedly smaller than that in the non-pregnancy group. With the increase of age, the levels of FT4 and TSH tended to be reduced, but the TSH level was increased to a certain degree in patients aged over 40 years. There were differences in the FT4 and TSH reference intervals during pregnancy in comparison among different regions and different detection methods. Region-specific reference intervals need to be established
\end{abstract}

Correspondence to: Dr Chunling Ma, Clinical Laboratory Department, Shandong Medical College, 1 Jucai Liu Road, Lanshan, Linyi, Shandong 276000, P.R. China

E-mail: ncs2pd@163.com; mach12003@163.com

${ }^{*}$ Contributed equally

Key words: free thyroxine, thyroid stimulating hormone, thyroid peroxidase antibody, pregnancy for different trimesters of pregnancy and different detection methods, which is conducive to accurate clinical judgment of thyroid function during pregnancy. TPOAb monitoring needs to be strengthened in the third trimester of pregnancy, and attention should be paid to the changes in thyroid function in pregnant women aged over 40 years.

\section{Introduction}

During pregnancy, changes in the levels of hormones such as estrogen and human chorionic gonadotropin (HCG) and the increased excretion of iodine in the kidney result in changes in thyroid hormones in pregnant women to varying degrees (1). Previous findings showed that the levels of free thyroxine (FT4) and thyroid stimulating hormone (TSH) during pregnancy are obviously different from those during non-pregnancy $(2,3)$. The application of non-pregnancy reference intervals lead to false detection rates to different degrees.

In the Guidelines for Diagnosis and Treatment of Pregnancy and Postpartum Thyroid Diseases stipulated by the American Thyroid Association (ATA) in 2011 (4) and the Chinese Society of Endocrinology and Chinese Society of Perinatal Medicine of the Chinese Medical Association in 2012 (2), it was proposed that the specific reference interval of thyroid hormones during pregnancy needs to be established. In recent years, relevant research has been conducted in many regions in China (5-10), but no unified standard has been formed, which repeatedly confirms the necessity for establishing region- and methodspecific reference intervals. Therefore, a statistical analysis was carried out in the present study for the detection results of thyroid hormones in healthy pregnant women in Linyi region, so as to establish a method-specific thyroid hormone reference interval during pregnancy in this region and provide a basis for accurate clinical diagnosis and treatment.

\section{Patients and methods}

Study objects. A total of 22,235 healthy pregnant women receiving examinations during pregnancy from October 2016 to October 2017 in the Women and Children's Health Care 


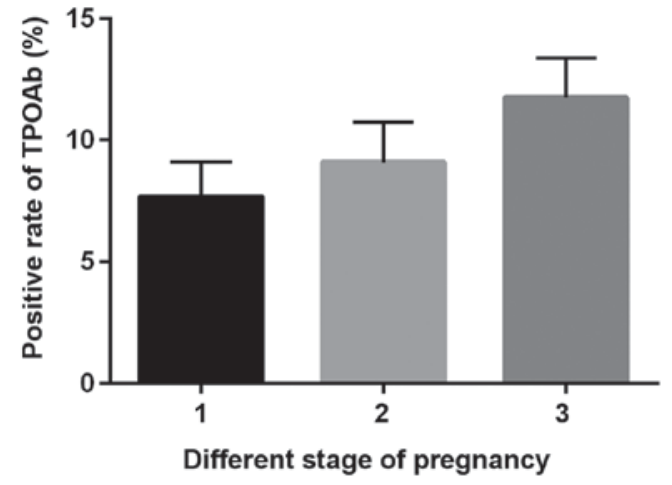

Figure 1. SPSS 19.0 software was used to plot different statistical graphs of the positive rate of TPOAb in different trimesters of pregnancy. Statistics reveal that the positive rate of TPOAb is increased with the prolonged trimester of pregnancy. Positive TPOAb is detected in a total of 873 pregnant women in the first trimester of pregnancy (7.67\%), 791 pregnant women in the second trimester of pregnancy $(9.09 \%)$, and 253 pregnant women in the third trimester of pregnancy $(11.74 \%)$. 1, First trimester of pregnancy; 2 , second trimester of pregnancy; 3 , third trimester of pregnancy.

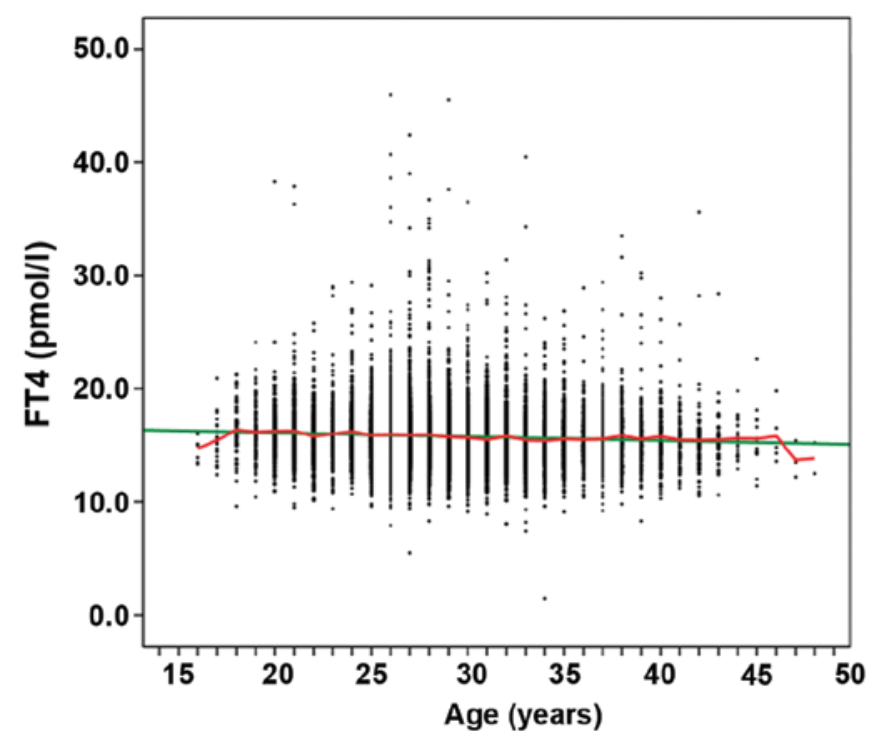

Figure 2. SPSS 19.0 software was used to draw a scatter plot of the correlation between FT4 and age in the first trimester of pregnancy, and a linear correlation analysis was conducted $\left(r^{2}=0.004\right)$. In this plot, the green line is a linear fitting line, and the red line represents the average of the age data. Statistics reveal that the detection results of FT4 in the first trimester of pregnancy present a skewed distribution.

Hospital of Linyi (Linyi, China) were selected, including 11,382 patients in the first trimester of pregnancy (within 12 weeks of pregnancy), 8,698 patients in the second trimester of pregnancy (13-27 weeks of pregnancy) and 2,155 patients in the third trimester of pregnancy (more than 28 weeks of pregnancy). The study patients were of 16-48 years of age and were divided to 6 groups according to their age: $\leq 20$ years group, 21-25 years group, 26-30 years group, 31-35 years group, 36-40 years group and $>40$ years group. Exclusion criteria for the study were: i) patients with a family history and past history of thyroid-related diseases, ii) patients taking iodine-containing drugs or drugs affecting thyroid function (except estrogens), iii) patients with palpable goiters, or iv) patients with autoimmune diseases. At the same time,

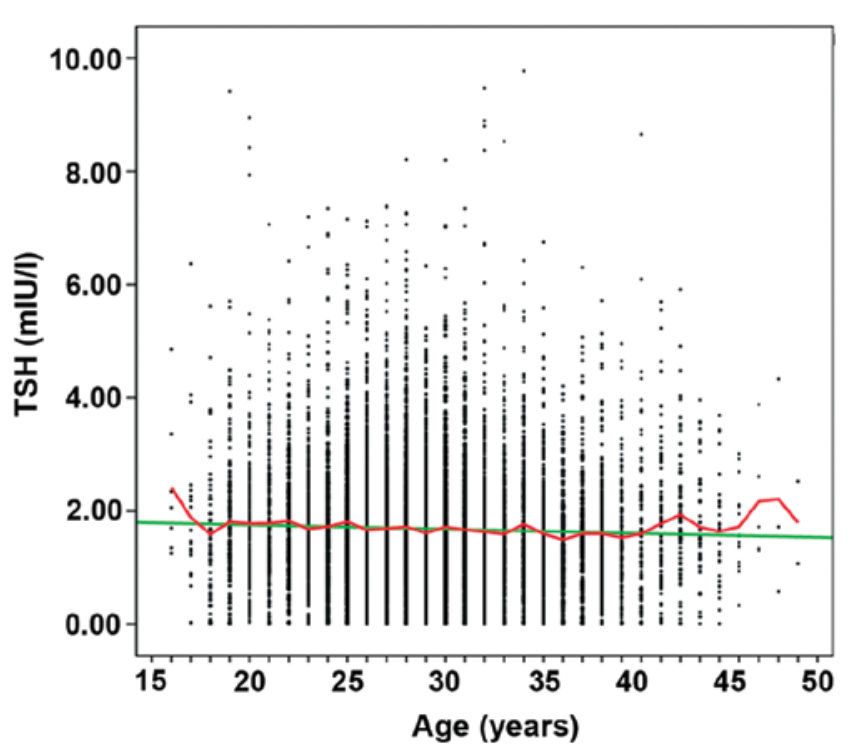

Figure 3. A scatter plot of the relationship between TSH and age in the first trimester of pregnancy was drawn via SPSS 19.0 software, and a linear correlation analysis was carried out $\left(r^{2}=0.001\right)$. In this plot, the green line is a linear fitting line, and the red line represents the average of the age data. Statistics revealed that the detection results of TSH in the first trimester of pregnancy present a skewed distribution.

990 healthy non-pregnant women during the same period undergoing thyroid hormone detection were selected as the control group. The study was approved by the Ethics Committee of Women and Children's Health Care Hospital of Linyi. Patients who participated in this study had complete clinical data. Signed informed consents were obtained from the patients or guardians.

Detection methods. Fasting venous blood $(3 \mathrm{ml})$ was collected in the morning and placed in a yellow vacuum tube containing coagulants. The two were inverted and mixed 5-8 times. After standing at room temperature for $30 \mathrm{~min}$, the mixture was centrifuged at $1,680 \mathrm{x}$ for $5 \mathrm{~min}$ at $25^{\circ} \mathrm{C}$ to separate the serum for detection on BY-320A centrifuge (Beijing Baiyang Medical Devices Co., Ltd., Beijing, China). The levels of FT4, TSH and thyroid peroxidase antibody (TPOAb) were detected via a Roche E601 automatic electrochemiluminescence analyzer. All reagents were provided by Roche.

Statistical analysis. Statistical Product and Service Solutions (SPSS) 19.0 (IBM Corp., Beijing, China) and Excel software were employed for statistical analysis. Data in each trimester of pregnancy were expressed as median and 95\% bilateral limiting values. Enumeration data were described by frequency and percentage. Chi-square test was used for the comparison between groups. $\mathrm{P}<0.05$ was considered to indicate a statistically significant difference.

\section{Results}

Comparison of the positive rates of TPOAb in healthy pregnant women in different pregnancy trimesters. Statistical detection revealed a total of 873 pregnant women with positive TPOAb in the first trimester of pregnancy, and the positive rate was 


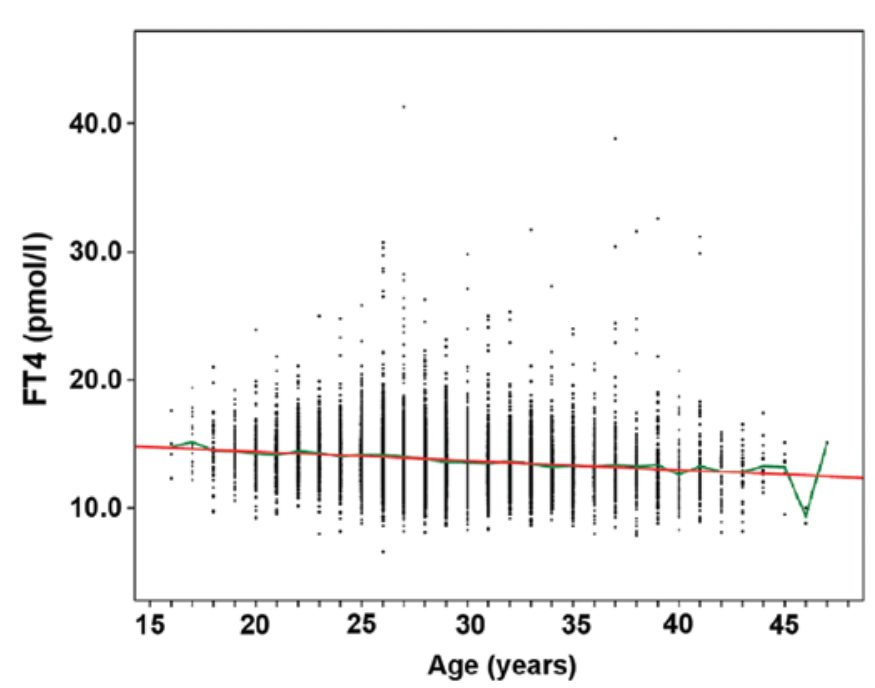

Figure 4. SPSS 19.0 software was employed to draw a scatter plot of the correlation between FT4 and age in the second trimester of pregnancy. In this plot, the red line is a linear fitting line, and the green line represents the average of the age data. Statistics show that the detection results of FT4 in the second trimester of pregnancy present a skewed distribution.

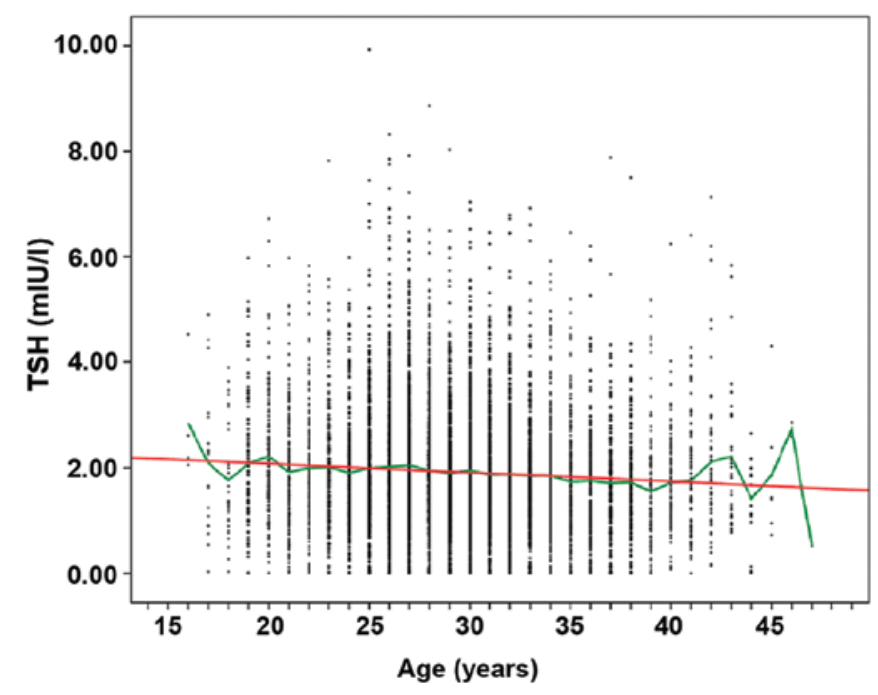

Figure 5. A scatter plot of the correlation between TSH and age in the second trimester of pregnancy was drawn via SPSS 19.0 software. In this plot, the red line is a linear fitting line, and the green line represents the average of the age data. Statistics reveal that the detection results of TSH in the second trimester of pregnancy present a skewed distribution.

7.67\%. A total of 791 pregnant women with positive TPOAb in the second trimester of pregnancy were detected, with a positive rate of $9.09 \%$. Positive TPOAb was detected in a total of 253 pregnant women in the third trimester of pregnancy, with a positive rate of $11.74 \%$. With the prolongation of pregnancy, the positive rate of TPOAb increased, and the positive rate in the third trimester was significantly higher than that in the first and second trimesters of pregnancy $(\mathrm{P}<0.05)$. TPOAb $\geq 34$ IU/1 set by Roche reagent specifications was considered positive (Fig. 1).

Analysis of the correlation of FT4 and TSH detection results in healthy pregnant women according to age. According to

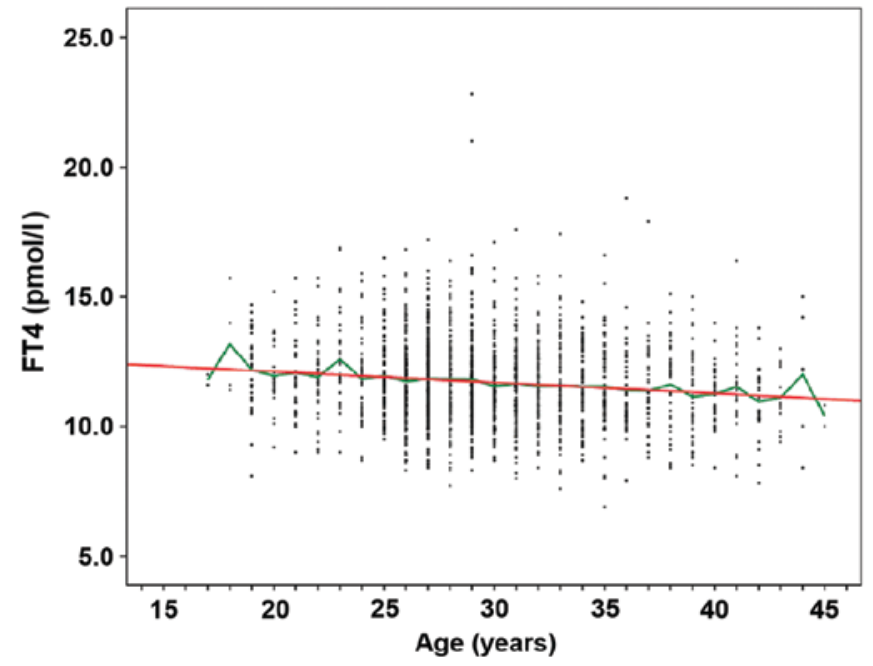

Figure 6. SPSS 19.0 software was employed to draw a scatter plot of the correlation between FT4 and age in the third trimester of pregnancy. In this plot, the red line is a linear fitting line, and the green line represents the average of the age data. Statistics reveal that the detection results of FT4 in the third trimester of pregnancy present a skewed distribution.

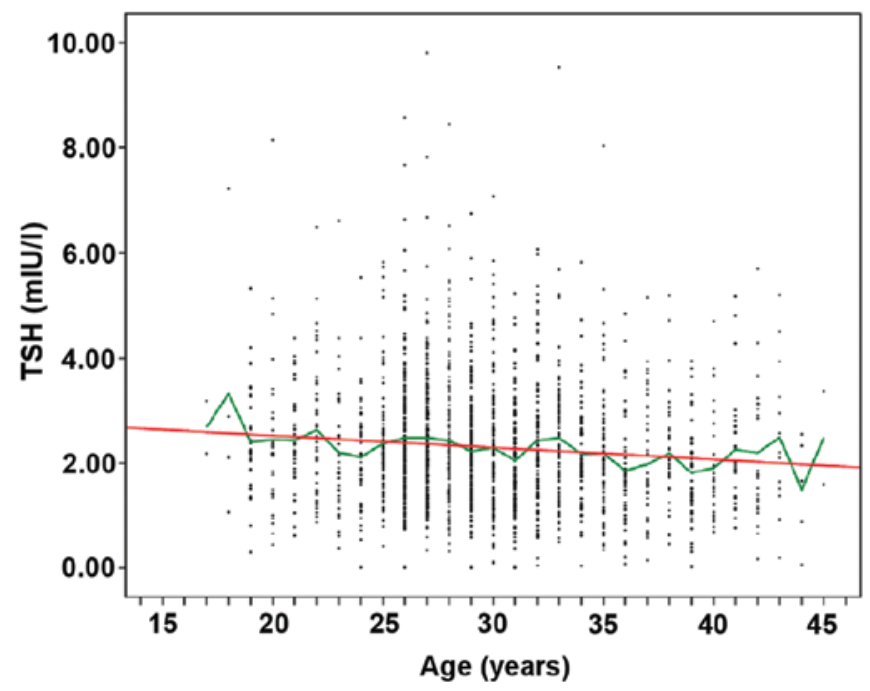

Figure 7. A scatter plot of the correlation between TSH and age in the third trimester of pregnancy was drawn using SPSS 19.0 software. In this plot, the red line is a linear fitting line, and the green line represents the average of the age data. Statistics reveal that the detection results of TSH in the third trimester of pregnancy present a skewed distribution.

the guideline (2), after patients with positive TPOAb were excluded, the remaining specimens included 10,509 cases in the first trimester of pregnancy, 7,907 cases in the second trimester of pregnancy, and 1,902 cases in the third trimester of pregnancy. FT4 and TSH detected data were statistically analyzed (Figs. 2-7).

Comparison of the levels of FT4 in different age groups in the first, second and third trimesters of pregnancy. The levels of FT4, TSH, and TPOAb were detected using a Roche E601 automatic electrochemiluminescence analyzer, and all reagents were provided by Roche. Statistical methods included SPSS 19.0 and Excel software, which were applied for 

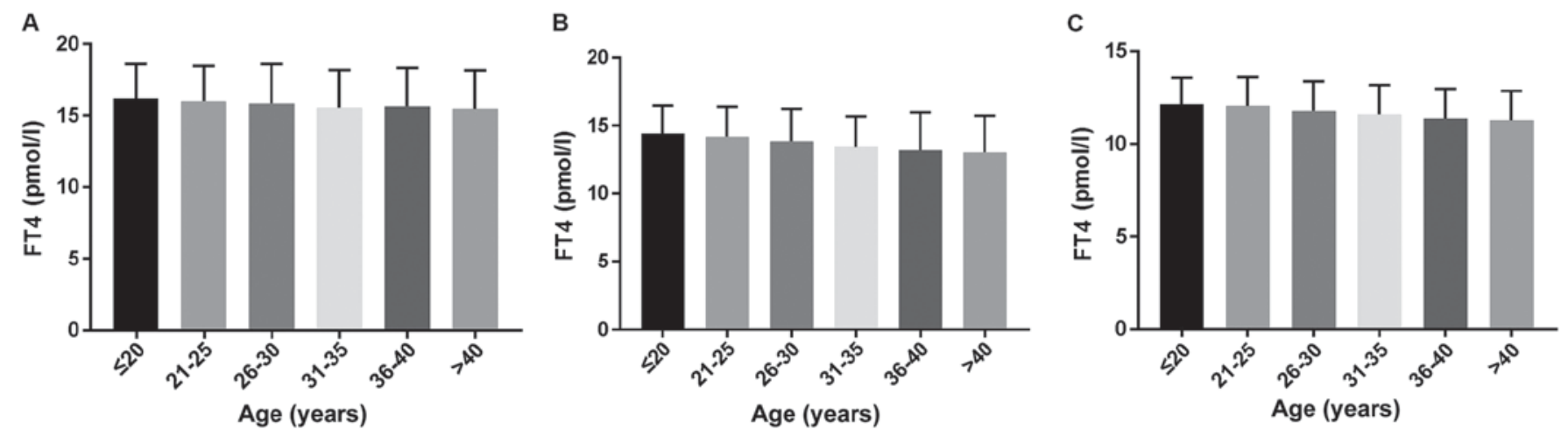

Figure 8. Comparison of the levels of FT4 in different age groups. (A) First trimester of pregnancy, (B) second trimester of pregnancy, (C) third trimester of pregnancy.
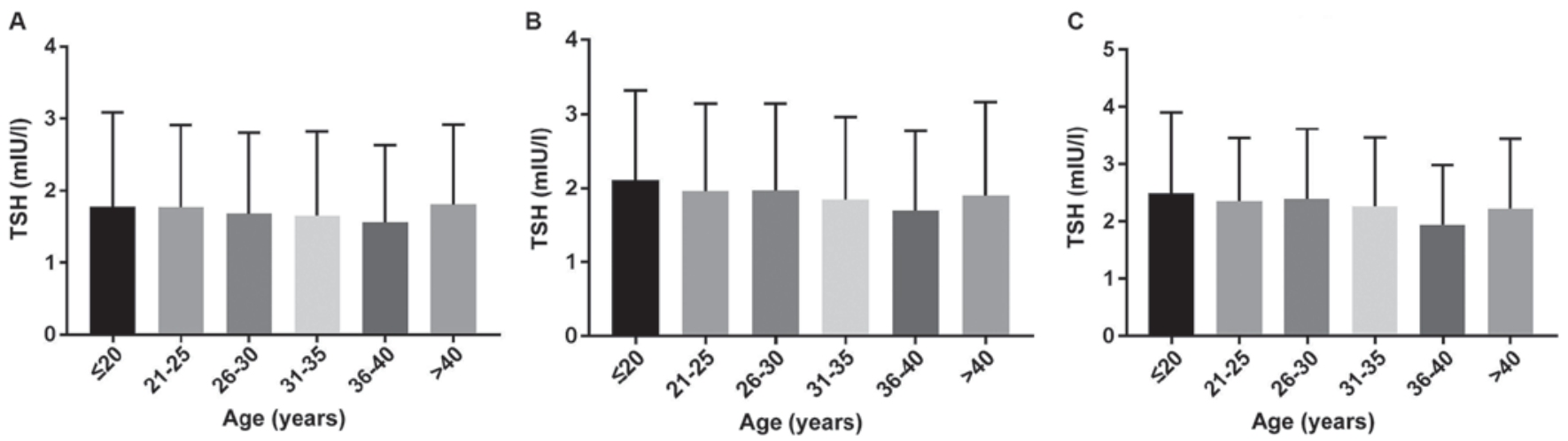

Figure 9. Comparison of the levels of TSH in different age groups. (A) First trimester of pregnancy, (B) second trimester of pregnancy, (C) third trimester of pregnancy.

statistical analysis. The median FT4 decreased gradually with increase of age in the second and third trimester of pregnancy $(\mathrm{P}<0.05)$ (Fig. 8).

Comparison of the levels of TSH in different age groups in the first, second and third trimesters of pregnancy. The levels of TSH in different age groups in the first, second and third trimesters of pregnancy were compared, and the Roche E601 automatic electrochemiluminescence analyzer was used to detect the levels of FT4, TSH and TPOAb. The median value of TSH decreased in the first and second trimester of pregnancy, but the median of TSH in the group of $>40$ years of age was significantly higher than that in the group of 36-40 years of age $(\mathrm{P}<0.05)$ (Fig. 9).

Reference intervals of FT4 and TSH in healthy pregnant women in different trimesters of pregnancy. According to the guideline (2), after patients with positive TPOAb, there were 10,509 cases in the first trimester of pregnancy, 7,907 cases in the second trimester of pregnancy and 1,902 cases in the third trimester of pregnancy. Statistical analysis results of FT4 and TSH revealed that all the results presented a skewed distribution, and the corresponding reference intervals were represented by median and $95 \%$ bilateral limiting values. Specific data are shown in Table I. Table I also shows that with the prolongation of pregnancy, the FT4 level tended to be gradually decreased whereas the TSH level was gradually increased. FT4 levels in the second and third trimesters of pregnancy were markedly lower than that in the non-pregnancy, and the differences were statistically significant. The TSH level in the first trimester of pregnancy was notably lower than that in the non-pregnancy, with a statistically significant difference.

Comparison of the detection results of FT4 and TSH in pregnant women in different age groups. The pregnant women were divided into five groups according to their age: $\leq 20$-year group, 21-25-year group, 26-30-year group, 31-35-year group, 36-40-year group and $>40$-year group. Statistics revealed that the medians of FT4 in the second and third trimesters of pregnancy showed a gradual decrease in trend with age, and those of TSH in the first and second trimesters of pregnancy also exhibited a tendency to decline with age in the following groups aged below 40 years, but they were increased again in the $>40$-year group. In the first, second and third trimesters of pregnancy, the medians of TSH in the $>40$-year group were obviously higher than those in 36- to 40-year group, and the differences were statistically significant (Table II).

Comparison of the reference intervals of FT4 and TSH with those shown in relevant studies in China. The detection results of this study were compared with those in relevant studies in China (6-10), which demonstrated that the reference interval of FT4 was not obviously different from those in the first trimester 
Table I. Reference intervals of FT4 and TSH in pregnant women in different pregnancy trimesters in Linyi region.

\begin{tabular}{lrrr}
\hline Group & $\mathrm{n}$ & FT4 (pmol/l) & TSH (mIU/l) \\
\hline First-trimester pregnancy & 10509 & $15.6(11.5-21.5)^{\mathrm{b}}$ & $1.51(0.04-4.42)^{\mathrm{a}, \mathrm{b}}$ \\
Second-trimester pregnancy & 7907 & $13.9(9.9-18.7)^{\mathrm{a}}$ & $1.90(0.09-4.70)$ \\
Third-trimester pregnancy & 1902 & $11.6(8.8-15.2)^{\mathrm{a}}$ & $2.14(0.59-5.16)$ \\
Non-pregnancy & 990 & $15.5(11.9-20.2)$ & $1.94(0.57-4.90)$
\end{tabular}

${ }^{a} \mathrm{P}<0.05$ vs. non-pregnancy group, and ${ }^{\mathrm{b}} \mathrm{P}<0.05$ vs. third-trimester pregnancy group.

Table II. Correlation of FT4 and TSH medians with the age of pregnant women.

\begin{tabular}{|c|c|c|c|c|c|c|c|c|c|}
\hline \multirow[b]{2}{*}{ Group } & \multicolumn{3}{|c|}{ First-trimester pregnancy } & \multicolumn{3}{|c|}{ Second-trimester pregnancy } & \multicolumn{3}{|c|}{ Third-trimester pregnancy } \\
\hline & $\mathrm{n}$ & FT4 (pmol/l) & TSH (mIU/l) & $\mathrm{n}$ & FT4 (pmol/l) & TSH (mIU/l) & $\mathrm{n}$ & FT4 (pmol/l) & TSH (mIU/l) \\
\hline$\leq 20$ years & 436 & 16.14 & 1.78 & 292 & 14.41 & 2.11 & 65 & 12.13 & 2.49 \\
\hline 21-25 years & 1,895 & 16.00 & 1.77 & 1283 & 14.19 & 1.96 & 260 & 12.03 & 2.35 \\
\hline $26-30$ years & 4,823 & 15.85 & 1.68 & 3619 & 13.84 & 1.97 & 847 & 11.76 & 2.39 \\
\hline $31-35$ years & 2,164 & 15.54 & 1.65 & 1782 & 13.44 & 1.84 & 458 & 11.58 & 2.26 \\
\hline $36-40$ years & 927 & 15.63 & $1.56^{\mathrm{a}}$ & 728 & 13.21 & $1.70^{\mathrm{a}}$ & 199 & 11.35 & $1.94^{\mathrm{a}}$ \\
\hline$>40$ years & 264 & 15.46 & 1.80 & 203 & 13.05 & 1.90 & 73 & 11.27 & 2.22 \\
\hline
\end{tabular}

${ }^{\mathrm{a}} \mathrm{P}<0.05$ vs. $>40$-year group.

Table III. Comparison of FT4 detection results in different regions via different methods.

\begin{tabular}{llllll}
\hline Region & Instrument & $\begin{array}{c}\text { First-trimester } \\
\text { pregnancy }\end{array}$ & $\begin{array}{c}\text { Second-trimester } \\
\text { pregnancy }\end{array}$ & $\begin{array}{c}\text { Third-trimester } \\
\text { pregnancy }\end{array}$ & Non-pregnancy \\
\hline Shenzhen & Roche & $17.56(11.65-22.16)^{\mathrm{a}}$ & $14.16(9.49-20.14)$ & $12.38(10.13-15.88)^{\mathrm{a}}$ & $18.71(14.35-21.69)^{\mathrm{a}}$ \\
Urumqi & Roche & $14.30(9.94-20.21)^{\mathrm{a}}$ & $13.52(9.69-17.77)$ & $10.92(7.28-15.99)^{\mathrm{a}}$ & $13.91(10.09-19.72)^{\mathrm{a}}$ \\
Jinan & Roche & $15.39(12.60-18.94)$ & $12.46(8.74-17.23)^{\mathrm{a}}$ & $11.45(8.64-17.21)$ & $14.62(11.06-18.59)^{\mathrm{a}}$ \\
Zibo & Beckman & $11.10(8.71-15.86)^{\mathrm{a}}$ & $9.31(5.98-12.22)^{\mathrm{a}}$ & $8.05(5.22-10.92)^{\mathrm{a}}$ & $12.12(7.86-14.41)^{\mathrm{a}}$ \\
Henan & Siemens & $11.07(9.55-12.59)^{\mathrm{a}}$ & $9.31(7.89-10.73)^{\mathrm{a}}$ & $8.16(6.86-9.46)^{\mathrm{a}}$ & $12.81(11.29-14.33)^{\mathrm{a}}$ \\
Linyi & Roche & $15.6(11.5-21.5)$ & $13.9(9.9-18.7)$ & $11.6(8.8-15.2)$ & $15.5(11.9-20.2)$ \\
\hline
\end{tabular}

${ }^{a} \mathrm{P}<0.05$ vs. Linyi region.

of pregnancy in Jinan, in the second trimester of pregnancy in Urumqi and Shenzhen, and in the third trimester of pregnancy in Jinan, but significantly different from those in other groups $(\mathrm{P}<0.05)$. In addition, there were no obvious differences in the reference interval of TSH in comparison with those in the first trimester of pregnancy in Jinan and in the second trimester of pregnancy in Urumqi, but significant differences were found in comparison with other groups $(\mathrm{P}<0.05)$. Specific results are shown in Tables III and IV.

\section{Discussion}

During pregnancy, thyroid dysfunction can lead to miscarriage, thyroid crisis, pregnancy-induced hypertension, fetal distress, intrauterine growth retardation and a series of maternal and fetal adverse reactions (1). In particular, maternal hypothyroidism during pregnancy can cause fetal developmental disorders in neural intelligence, thus bringing serious burdens to family and the society. Thus, it is crucial to maintain maternal normal thyroid function during pregnancy. A series of compensatory changes occur in maternal thyroid glands due to hormone and immunophysiological changes during pregnancy, so the thyroid function indexes during pregnancy are quite different from those during non-pregnancy. Therefore, the application of reference intervals of thyroid function indexes during pregnancy may lead to misdiagnosis and missed diagnosis to a certain degree (3), and it is necessary to establish a reference interval for specific thyroid function during pregnancy. In the guideline stipulated in China (2), it is recommended to apply FT4, TSH and TPOAb as indexes of thyroid screening 
Table IV. Comparison of TSH detection results in different regions via different methods.

\begin{tabular}{llllll}
\hline Region & Instrument & $\begin{array}{c}\text { First-trimester } \\
\text { pregnancy }\end{array}$ & $\begin{array}{c}\text { Second-trimester } \\
\text { pregnancy }\end{array}$ & $\begin{array}{c}\text { Third-trimester } \\
\text { pregnancy }\end{array}$ & Non-pregnancy \\
\hline Shenzhen & Roche & $1.10(0.23-3.58)^{\mathrm{a}}$ & $2.07(0.13-3.96)^{\mathrm{a}}$ & $2.62(1.08-3.62)^{\mathrm{a}}$ & $2.63(1.00-5.14)^{\mathrm{a}}$ \\
Urumqi & Roche & $1.85(0.06-4.80)^{\mathrm{a}}$ & $1.89(0.29-5.84)$ & $2.57(0.65-7.64)^{\mathrm{a}}$ & $2.09(0.48-4.56)^{\mathrm{a}}$ \\
Jinan & Roche & $1.52(0.27-4.27)$ & $2.39(0.53-4.75)^{\mathrm{a}}$ & $2.52(0.76-4.59)^{\mathrm{a}}$ & $2.68(0.83-4.79)^{\mathrm{a}}$ \\
Zibo & Beckman & $1.78(0.22-4.53)^{\mathrm{a}}$ & $2.14(0.42-4.88)^{\mathrm{a}}$ & $2.40(0.54-5.36)^{\mathrm{a}}$ & $2.67(0.66-4.60)^{\mathrm{a}}$ \\
Henan & Siemens & $1.68(0.65-2.71)^{\mathrm{a}}$ & $2.03(0.96-3.10)^{\mathrm{a}}$ & $2.31(1.06-3.56)^{\mathrm{a}}$ & $2.35(1.21-3.49)^{\mathrm{a}}$ \\
Linyi & Roche & $1.51(0.04-4.42)$ & $1.90(0.09-4.70)$ & $2.14(0.59-5.16)$ & $1.94(0.57-4.90)$ \\
\hline
\end{tabular}

${ }^{\mathrm{a}} \mathrm{P}<0.05$ vs. Linyi region.

during pregnancy. The guideline suggests that the positive diagnostic criteria of TPOAb is higher than the upper limit of the reference value provided by the kit. Therefore, statistical analyses were mainly conducted for reference intervals of the two indexes, FT4 and TSH, in this study.

$\mathrm{TPOAb}$ is produced by the release of thyroid peroxidase (TPO) from the follicles of thyroid glands into the blood to stimulate the body's immune system, thereby reducing the production of thyroid hormones and stimulating TSH secretion. Positive TPOAb often indicates the presence of thyroid damage and may increase the risks of miscarriage and premature delivery. Reports worldwide have demonstrated that the positive rate of TPOAb during pregnancy is in the range of $9.17-17.58 \%$ (11-13). However, in this study, the positive rates of TPOAb in the first, second and third trimesters of pregnancy were $7.67,9.09$ and $11.74 \%$, respectively, with the overall positive rate of $8.62 \%$, which was slightly lower than results in other reports. This may be related to the detection method, the iodine status of the region, and the number of the detected individuals. This study showed that the TPOAb positive rate in the third trimester of pregnancy is considerably higher than that in the first trimester of pregnancy, indicating a statistically significant difference. For pregnant women in the third trimester of pregnancy, monitoring of TPOAb should be strengthened. It was also found in this study that the FT4 level in the third trimester is remarkably lower than that in the first trimester pregnancy, while the TSH level in the third trimester of pregnancy was notably higher than that in the first trimester of pregnancy, suggesting that the probability of hypothyroidism in pregnant women in the third trimester of pregnancy is greater, which needs particular attention.

The present study revealed that with the prolongation of pregnancy, the FT4 level exhibited a gradually decreasing trend while the TSH level had a gradually increasing trend, which is consistent with the research findings of other scholars (6-11). The FT4 level in the third trimester of pregnancy was significantly lower than that in the first trimester. The underlying cause may be that the increased excretion rate of iodine in the kidney of pregnant women in the third trimester of pregnancy and the increased demand for iodine in the fetus result in a relative lack of maternal iodine (14), thus leading to the decreased production of thyroid hormones. TSH level in the first trimester of pregnancy was obviously lower than that in the third trimester of pregnancy. This is mainly because HCG peaks at 8-10 weeks of pregnancy and then continues to decline, the alpha subunits of HCG and TSH are similar, and the negative feedback regulation leads to a marked decrease in the TSH level in the first trimester of pregnancy, which is then gradually increased. In the second and third trimesters of pregnancy, the FT4 level tended to decrease with the increase of the age of pregnant woman, which is similar to the results of Kuo et al (15) and Ademuyiwa et al (16). This may be related to the hyposecretion of hormones with the increase of age (16), but the specific reason has yet to be determined. The changed trend in TSH in this study is contrary to that in the study of Ademuyiwa et al (16). In this study, it was found that TSH exhibited a tendency to decline with the increase of age in women aged $<40$ years, and it only tended to be increased in the $>40$-year group, which might be due to the different research groups and the number of included patients. In the 40-year group, the FT4 level was the lowest among all age groups, while the TSH level was markedly higher than that in 35- to 40-year group, suggesting that the occurrence probability of hypothyroidism is higher in the 40-year group.

Recent studies worldwide have proposed that region- and method-specific reference intervals for thyroid function during pregnancy need to be established for self-serving populations in various prenatal screening centers $(5,17)$. Comparisons of study results of related institutions in China (6-10) also verify this view. Using the same detection method, significant differences were found in FT4 and TSH levels in groups in Linyi except FT4 levels in the first and third trimesters of pregnancy and TSH level in the first trimester of pregnancy compared with those in Jinan. Compared with those in Urumqi, significant differences were detected in FT4 and TSH levels in all groups except FT4 and TSH levels in the second trimester of pregnancy. Compared with those in Shenzhen, there were notable differences in FT4 and TSH levels in all the groups except FT4 level in the second trimester of pregnancy $(\mathrm{P}<0.05)$. Detection results were notably different among different detection methods regardless of the region $(\mathrm{P}<0.05)$. Therefore, it is imperative to establish region- and method-specific thyroid hormone reference intervals during pregnancy.

In summary, thyroid hormones during pregnancy vary with pregnancy, region, and detection methods. Each region 
or medical institution should establish its own specific thyroid hormone reference intervals during pregnancy to provide accurate diagnostic criteria for the region or medical institution. Thyroid hormones during pregnancy change with age and pregnancy. For pregnant women aged over 40 years in the third trimester of pregnancy, thyroid hormone levels need intensive monitoring.

\section{Acknowledgements}

Not applicable.

\section{Funding}

This study was supported by the Science and Technology Development and Innovation Project in Linyi (contract no. 201717050).

\section{Availability of data and materials}

The datasets used and/or analyzed during the present study are available from the corresponding author on reasonable request.

\section{Authors' contributions}

QZ and YZ collected the data of patents and extracted venous blood. JZ and XY recorded and interpreted FT4 level. YH and HL analyzed TSH levels. QZ, YZ and CM were responsible for the statistical analysis. All authors read and approved the final manuscript.

\section{Ethics approval and consent to participate}

The study was approved by the Ethics Committee of Women and Children's Health Care Hospital of Linyi (Linyi, China). Patients who participated in this study had complete clinical data. Signed informed consents were obtained from the patients or guardians.

\section{Patient consent for publication}

Not applicable.

\section{Competing interests}

The authors declare that they have no competing interests.

\section{References}

1. Teng WP and Shan ZY: Confusion and thought of the diagnosis and treatment for thyroid diseases in pregnancy. Zhonghua Nei Ke Za Zhi 51: 1-4, 2012 (In Chinese).

2. Alexander EK, Pearce EN, Brent GA, Brown RS, Chen H, Dosiou C, Grobman WA, Laurberg P, Lazarus JH, Mandel SJ, et al: 2017 Guidelines of the American Thyroid Association for the diagnosis and management of thyroid disease during pregnancy and the postpartum. Thyroid 27: 315-389, 2017.
3. Stricker R, Echenard M, Eberhart R, Chevailler MC, Perez V, Quinn FA and Stricker R: Evaluation of maternal thyroid function during pregnancy: The importance of using gestational agespecific reference intervals. Eur J Endocrinol 157: 509-514, 2007.

4. Stagnaro-Green A, Abalovich M, Alexander E, Azizi F, Mestman J, Negro R, Nixon A, Pearce EN, Soldin OP, Sullivan S, et al; American Thyroid Association Taskforce on Thyroid Disease During Pregnancy and Postpartum: Guidelines of the American Thyroid Association for the diagnosis and management of thyroid disease during pregnancy and postpartum. Thyroid 21: 1081-1125, 2011.

5. Wang QW, Yu B, Huang RP, Cao F, Zhu ZQ, Sun DC and Zhou H: Assessment of thyroid function during pregnancy: The advantage of self-sequential longitudinal reference intervals. Arch Med Sci 7: 679-684, 2011.

6. Yu B, Wang QW, Huang RP, Cao F, Zhu ZQ, Sun DC, Zhou H and Zhang YM: Establishment of self-sequential longitudinal reference intervals of maternal thyroid function during pregnancy. Exp Biol Med (Maywood) 235: 1212-1215, 2010.

7. Duan Y, Peng L, Cui Y and Jiang Y: Reference intervals for thyroid function and the negative correlation between FT4 and HbA1c in pregnant women of West China. Clin Lab 61: 777-783, 2015.

8. Zhang X, Yao B, Li C, Mao J, Wang W, Xie X, Teng X, Han C, Zhou W, Li C, et al: Reference intervals of thyroid function during pregnancy: Self-sequential longitudinal study versus cross-sectional study. Thyroid 26: 1786-1793, 2016.

9. Zhang J, Li W, Chen QB, Liu LY, Zhang W, Liu MY, Wang YT, Li WY and Zeng LZ: Establishment of trimester-specific thyroid stimulating hormone and free thyroxine reference interval in pregnant Chinese women using the Beckman Coulter UniCel ${ }^{\mathrm{TM}}$ DxI 600. Clin Chem Lab Med 53: 1409-1414, 2015.

10. Wang D, Li D, Guo X, Yu S, Qiu L, Cheng X, Xu T, Li H and Liu H: Effects of sex, age, sampling time, and season on thyroidstimulating hormone concentrations: A retrospective study. Biochem Biophys Res Commun 506: 450-454, 2018.

11. Gao X, Li Y, Li J, Liu A, Sun W, Teng W and Shan Z: Gestational TSH and FT4 reference intervals in Chinese women: A systematic review and meta-analysis. Front Endocrinol (Lausanne) 9: 432, 2018.

12. Sarkhail P, Mehran L, Askari S, Tahmasebinejad Z, Tohidi M and Azizi F: Maternal thyroid function and autoimmunity in 3 trimesters of pregnancy and their offspring's thyroid function. Horm Metab Res 48: 20-26, 2016.

13. Thangaratinam S, Tan A, Knox E, Kilby MD, Franklyn J and Coomarasamy A: Association between thyroid autoantibodies and miscarriage and preterm birth: Meta-analysis of evidence. BMJ 342: d2616, 2011.

14. Shan Z, Chen L, Lian X, Liu C, Shi B, Shi L, Tong N, Wang S, Weng J, Zhao J, et al: Iodine status and prevalence of thyroid disorders after introduction of mandatory universal salt iodization for 16 years in China: A cross-sectional study in 10 cities. Thyroid 26: 1125-1130, 2016.

15. Kuo FC, Su SW, Wu CF, Huang MC, Shiea J, Chen BH, Chen YL and Wu MT: Relationship of urinary phthalate metabolites with serum thyroid hormones in pregnant women and their newborns: A prospective birth cohort in Taiwan. PLoS One 10: e0123884, 2015.

16. Ademuyiwa O, Odusoga OL, Adebawo OO and Ugbaja R: Endogenous antioxidant defences in plasma and erythrocytes of pregnant women during different trimesters of pregnancy. Acta Obstet Gynecol Scand 86: 1175-1182, 2007.

17. Veltri F, Belhomme J, Kleynen P, Grabczan L, Rozenberg S, Pepersack T and Poppe K: Maternal thyroid parameters in pregnant women with different ethnic backgrounds: Do ethnicityspecific reference ranges improve the diagnosis of subclinical hypothyroidism? Clin Endocrinol (Oxf) 86: 830-836, 2017.

This work is licensed under a Creative Commons Attribution-NonCommercial-NoDerivatives 4.0 International (CC BY-NC-ND 4.0) License. 